\title{
Editorial: Less and Non-invasive Hemodynamic Monitoring Techniques
}

\author{
Bernd Saugel ${ }^{1 *}$ and Samir G. Sakka ${ }^{2}$ \\ ${ }^{1}$ Department of Anesthesiology, Center of Anesthesiology and Intensive Care Medicine, University Medical Center \\ Hamburg-Eppendorf, Hamburg, Germany, ${ }^{2}$ Department of Anesthesiology and Operative Intensive Care Medicine, University \\ of Witten/Herdecke, Cologne Merheim Medical Center, Cologne, Germany
}

Keywords: cardiovascular dynamics, cardiac output, blood pressure, intensive care medicine, anesthesiology, goal-directed therapy

\section{Editorial on the Research Topic}

\section{Less and Non-invasive Hemodynamic Monitoring Techniques}

The measurement or estimation of hemodynamic variables reflecting blood pressure, blood flow, cardiac contractility, cardiac preload, and cardiac afterload plays a pivotal role in the monitoring, diagnostic workup, and treatment of critically ill patients treated in the intensive care unit or in patients having major surgery.

Besides pulmonary artery catheterization that is established as a classical method to observe, measure, and derive a variety of variables reflecting cardiovascular and oxygen dynamics $(1,2)$ a variety of "modern" less- and non-invasive hemodynamic monitoring methods became available during the last decades $(3,4)$. The physical measurement principles, clinical applications, and limitations of these technologies are discussed in a series of articles that are part of the research topic "Less- and Non-invasive Hemodynamic Monitoring Techniques."

As a very basic hemodynamic variable and as a main determinant of the organs' perfusion

Edited and reviewed by: Ata Murat Kaynar,

University of Pittsburgh, United States

${ }^{*}$ Correspondence:

Bernd Saugel

bernd.saugel@gmx.de

Specialty section:

This article was submitted to Intensive Care Medicine and

Anesthesiology,

a section of the journal

Frontiers in Medicine

Received: 29 June 2018

Accepted: 29 August 2018

Published: 19 September 2018

Citation:

Saugel B and Sakka SG (2018)

Editorial: Less and Non-invasive Hemodynamic Monitoring Techniques.

Front. Med. 5:258.

doi: 10.3389/fmed.2018.00258 pressure, arterial blood pressure is part of routine monitoring in intensive care medicine and anesthesiology. In a narrative review article, methods for non-invasive intermittent and continuous blood pressure monitoring are summarized (Meidert and Saugel). The authors recommend monitoring of blood pressure with intermittent oscillometry in hemodynamically stable, lowrisk patients. In surgical patients at risk for hemodynamic instability, continuous non-invasive blood pressure monitoring with innovative techniques might become an option in the near future. In critically ill and high-risk surgical patients, continuous invasive blood pressure monitoring with an arterial catheter will be the method of choice in the foreseeable future. Based on these general recommendations, an article by Stenglova and Benes describes the evidence for the use of continuous non-invasive blood pressure monitoring during surgery and its potential to improve postoperative outcome by an early recognition (or even prediction) of hypotension.

In addition to blood pressure, the analysis of the arterial blood pressure waveform (pulse wave analysis) enables stroke volume, cardiac output, and dynamic cardiac preload parameters to be assessed using invasive (arterial catheter) or non-invasive (finger cuff) methods. Several articles discuss pulse wave analysis and its use in clinical practice.

Grensemann comprehensively explains the basic measurement principle of commercially available invasive monitoring systems using pulse wave analysis to estimate cardiac output. He emphasizes that pulse wave analysis is limited in patients with altered vascular tone and that uncalibrated systems should be used to follow cardiac output changes (trend monitoring) rather than to guide therapy based on absolute values of cardiac out. A review article by Yamada et al. 
describes how minimally invasive and non-invasive hemodynmaic monitoring techniques can be used to guide perioperative hemodynamic therapy and eventually improve postoperative outcome in surgical patients. The authors conclude that "monitoring equipment that can provide precise hemodynamic information without the complications and complexity of invasive techniques can facilitate individualized hemodynamic management and lead to improved outcomes."

In addition to this narrative review, a perspective article by Saugel and Reuter focuses on the use of invasive uncalibrated pulse wave analysis for perioperative hemodynamic management (often referred to as "perioperative goal-directed therapy"). The article briefly summarizes the evidence and concludes that perioperative goal-directed therapy based on pulse wave analysis-derived blood flow and dynamic cardiac preload variables can improve patient outcome in high-risk patients. This conclusion is in line with the results of recent metaanalyses showing that perioperative goal-directed therapy seems to reduce postoperative morbidity $(5,6)$. However, further well-designed and adequately powered studies are needed to answer open questions about optimal target variables and values and about how to implement these perioperative treatment strategies in clinical routine. Another perspective article written by Nicklas and Saugel discusses current evidence and open research questions related to completely non-invasive hemodynamic monitoring methods for perioperative hemodynamic management. Nguyen and Squara provide an in-depth review of non-invasive methods to estimate cardiac output besides pulse wave analysis. They explain the basic measurement principles and validation data of bioimpedance/bioreactance, partial carbon dioxide rebreathing, pulse wave transit time, ultrasonic methods, and inductance thoracocardiography. In particular, the authors focus on the feasibility of these methods in the intensive care

\section{REFERENCES}

1. Gidwani UK, Mohanty B, Chatterjee K. The pulmonary artery catheter: a critical reappraisal. Cardiol Clin. (2013). 31:545-65. doi: 10.1016/j.ccl.2013.07.008

2. Cholley BP. Benefits, risks and alternatives of pulmonary artery catheterization. Curr Opin Anaesthesiol. (1998) 11:645-50.

3. Teboul JL, Saugel B, Cecconi M, De Backer D, Hofer CK, Monnet X, et al. Less invasive hemodynamic monitoring in critically ill patients. Intensive Care Med. (2016) 42:1350-9. doi: 10.1007/s00134-016-4375-7

4. Saugel B, Cecconi M, Wagner JY, Reuter DA. Noninvasive continuous cardiac output monitoring in perioperative and intensive care medicine. Br J Anaesth. (2015) 114:562-75. doi: 10.1093/bja/aeu447

5. Chong MA, Wang Y, Berbenetz NM, McConachie I. Does goal-directed haemodynamic and fluid therapy improve perioperative outcomes? a systematic review and meta-analysis. Eur J Anaesthesiol. (2018) 35:469-83. doi: 10.1097/EJA.000000000 0000778

6. Pearse RM, Harrison DA, MacDonald N, Gillies MA, Blunt M, Ackland G, et al. (2014) Effect of a perioperative, cardiac output-guided hemodynamic unit setting and emphasize that hemodynamic monitoring of critically ill patients requires good measurement performance in terms of accuracy, precision, and step-response change. They conclude that "further developments are needed to provide clinicians with sufficiently accurate devices for routine use."

Optimization of oxygen delivery to the end-organs is the ultimate goal of therapeutic interventions aiming at an optimization of global cardiovascular dynamics. Accordingly, Molnar and Nemeth emphasize that-in addition to global blood flow variables such as stroke volume or cardiac outputmarkers of tissue oxygenation need to be considered during resuscitation of patients with circulatory shock. They explain the (patho)physiology of oxygen delivery, consumption, and extraction and discuss the value of central venous oxygen saturation to individually tailor therapeutic interventions to the individual patient's needs. The authors advocate for multimodal and individualized hemodynamic treatment strategies which should integrate various physiological variables (e.g., central venous oxygen saturation, lactate, venous-to-arterial carbon dioxide gap).

In summary, this series of articles reflects that a variety of innovative less- and non-invasive methods for advanced hemodynamic monitoring in intensive care and perioperative medicine are currently available. Future research needs to confirm that goal-directed optimization of global hemodynamics based on advanced less- and non-invasive hemodynamic monitoring can eventually improve oxygen delivery and have beneficial impact on patient outcome.

\section{AUTHOR CONTRIBUTIONS}

BS and SGS drafted the manuscript and approved the final version of the manuscript to be published.

therapy algorithm on outcomes following major gastrointestinal surgery: a randomized clinical trial and systematic review. JAMA 311:2181-90. doi: 10.1001/jama.2014.5305

Conflict of Interest Statement: BS and SGS collaborate with Pulsion Medical Systems SE (Feldkirchen, Germany) as members of the medical advisory board and received honoraria for giving lectures and refunds of travel expenses from Pulsion Medical Systems SE. BS received research support from Edwards Lifesciences (Irvine, CA, USA). BS received institutional research grants, unrestricted research grants, and refunds of travel expenses from Tensys Medical Inc. (San Diego, CA, USA). BS received honoraria for giving lectures from CNSystems Medizintechnik AG (Graz, Austria). BS received refunds of travel expenses from CNSystems Medizintechnik AG (Graz, Austria).

Copyright (c) 2018 Saugel and Sakka. This is an open-access article distributed under the terms of the Creative Commons Attribution License (CC BY). The use, distribution or reproduction in other forums is permitted, provided the original author(s) and the copyright owner(s) are credited and that the original publication in this journal is cited, in accordance with accepted academic practice. No use, distribution or reproduction is permitted which does not comply with these terms. 